\title{
Incidence rate and clinical impacts of arrhythmia following COVID-19: a systematic review and meta-analysis of 17,435 patients
}

\author{
Shu-Chen Liao ${ }^{1,2 \dagger}{ }^{+}$Shih-Chieh Shao ${ }^{3,4+}{ }^{4}$ Chi-Wen Cheng ${ }^{2,5}$, Yung-Chang Chen ${ }^{2,6}$ and Ming-Jui Hung $2,57^{*}$
}

\begin{abstract}
Arrhythmia is a potential cardiovascular complication of Coronavirus Disease 2019 (COVID-19) [1]. In one case series of patients hospitalized with COVID-19, 16.7\% developed unspecified arrhythmia [2], while another case series indicated sustained ventricular tachycardia or ventricular fibrillation among $5.9 \%$ of patients hospitalized with COVID-19 [3]. However, incidence rates of arrhythmia and mortality rates after incident arrhythmia in COVID-19 patients have not been systematically established.

We searched for relevant studies cited in PubMed or Embase up to September 15, 2020, using the terms "COVID-19", "arrhythmia", "incidence", "mortality," and "prognosis" with suitable MeSH terms. All studies were selected and reviewed by two reviewers (SCL and SCS). The final list of included studies and data extractions were derived through extensive discussion with agreement from both authors. Statistical analyses were performed using MedCalc (Windows) version 15.0 (MedCalc Software, Ostend, Belgium). Outcomes were reported as proportions with $95 \%$ confidence interval (CI), based on the random effects model. The heterogeneity among studies was detected by the Cochran $Q$ test with $p$ value and the $I^{2}$ statistic.
\end{abstract}

\footnotetext{
*Correspondence: hmj1447@cgmh.org.tw

${ }^{\dagger}$ Shu-Chen Liao and Shih-Chieh Shao have contributed equally to this work

${ }^{2}$ College of Medicine, Chang Gung University, Taoyuan, Taiwan

${ }^{5}$ Section of Cardiology, Department of Internal Medicine, Keelung Chang Gung Memorial Hospital, No. 222, Maijin Rd., Anle Dist., Keelung, Taiwan

${ }^{7}$ Community Medicine Research Center, Keelung Chang Gung Memorial Hospital, Keelung, Taiwan

Full list of author information is available at the end of the article
}

Of 645 potential studies screened, we excluded 143 duplicate studies, 66 irrelevant studies, 12 conference abstracts, 241 other types of publications (e.g., preprints, protocols, opinions, recommendations, editorials, commentaries, retractions and reviews), 114 studies without incidence or mortality data, and 13 non-English studies. We included 56 studies from 11 countries comprising 17,435 patients with COVID-19. Study characteristics for included articles are listed in Table 1. Notably, most studies only included hospitalized patients with COVID-19 (96.4\%). The overall incidence of arrhythmia in COVID-19 patients was $16.8 \%$ (95\% CI: $12.8-21.2 \% ; I^{2}$ : $98.0 \%, p<0.001$ ) (Fig. 1a). The incidence of different types of arrhythmia in patients with COVID-19 was as follows: 12.0\% (22 studies, 95\% CI: 8.6-15.9\%) for non-classified arrhythmia, $8.2 \%$ (14 studies, 95\% CI: $5.5-11.3 \%$ ) for atrial fibrillation/atrial flutter/atrial tachycardia, $10.8 \%$ (26 studies, 95\% CI: 6.6-15.9\%) for conduction disorders, $8.6 \%$ (5 studies, $95 \%$ CI: 4.5-13.9\%) for premature contraction and 3.3\% (16 studies, 95\% CI: 1.9-4.9\%) for ventricular fibrillation/ventricular tachycardia. We found the mortality was $20.3 \%$ (95\% CI: $12.9-29.0 \% ; I^{2}: 72.8 \%$, $p<0.001)$ in COVID-19 patients who developed arrhythmia (Fig. 1b).

Compared to the incident arrhythmia in patients with community-acquired pneumonia $(4.7 \%$, 95\% CI: $2.4-$ 8.9) [4], the present study indicates higher incidence of arrhythmia in COVID-19 patients (16.8\%) with 2 out of 10 patients dying after developing arrhythmia. The possible mechanisms of arrhythmia may involve cardiac damage from metabolic disarray, hypoxia, neurohormonal or inflammatory stress and infection-related original author(s) and the source, provide a link to the Creative Commons licence, and indicate if changes were made. The images or other third party material in this article are included in the article's Creative Commons licence, unless indicated otherwise in a credit line to the material. If material is not included in the article's Creative Commons licence and your intended use is not permitted by statutory regulation or exceeds the permitted use, you will need to obtain permission directly from the copyright holder. To view a copy of this licence, visit http://creativecommons.org/licenses/by/4.0/. The Creative Commons Public Domain Dedication waiver (http://creativeco mmons.org/publicdomain/zero/1.0/) applies to the data made available in this article, unless otherwise stated in a credit line to the data. 
Table 1 Study characteristics

\begin{tabular}{|c|c|c|c|c|c|c|c|c|}
\hline Author/Year & Country & Study design & Setting & Male & Age & HF & CAD & Medication \\
\hline DuY/2020 & Wuhan/China & $\begin{array}{l}\text { Retrospective ( } 2 \text { cent- } \\
\text { ers) }\end{array}$ & Inpatient & 72.9 & $65.8^{\mathrm{a}}$ & NA & 11.8 & $\begin{array}{l}\text { Anti-influenza drugs: } \\
\text { 77.6; Lopinavir-Riton- } \\
\text { avir: } 12.9\end{array}$ \\
\hline Wang D/2020 & Wuhan/China & $\begin{array}{l}\text { Retrospective (1 } \\
\text { center) }\end{array}$ & Inpatient & 54.3 & 56.0 & NA & NA & $\begin{array}{l}\text { Anti-influenza drugs: } \\
\text { 89.9; Azithromycin: } \\
\text { 18.1 }\end{array}$ \\
\hline Guo T/2020 & Wuhan/China & $\begin{array}{l}\text { Retrospective (1 } \\
\text { center) }\end{array}$ & Inpatient & 48.7 & $58.5^{\mathrm{a}}$ & NA & 11.2 & $\begin{array}{l}\text { Anti-influenza drugs: } \\
88.8\end{array}$ \\
\hline Rosenberg ES/2020 & New York/USA & $\begin{array}{l}\text { Retrospective (multi- } \\
\text { center) }\end{array}$ & Inpatient & 59.7 & 63.0 & 6.7 & 12.0 & $\begin{array}{l}\text { Hydroxychloroquine: } \\
\text { 18.8; Azithromycin: } \\
\text { 14.7; Hydroxychloro- } \\
\text { quine + Azithromycin: } \\
51.1\end{array}$ \\
\hline Lei S/2020 & Wuhan/China & $\begin{array}{l}\text { Retrospective ( } 3 \text { cent- } \\
\text { ers) }\end{array}$ & Inpatient & 41.2 & 55.0 & NA & NA & Lopinavir-Ritonavir: 100 \\
\hline Saleh M/2020 & New York/USA & Prospective (3 centers) & Inpatient & 57.2 & $58.5^{\mathrm{a}}$ & 7.5 & 11.4 & $\begin{array}{l}\text { Hydroxychloroquine/ } \\
\text { Chloroquine: 40.8; } \\
\text { (Hydroxychloro- } \\
\text { quine/Chloro- } \\
\text { quine) + Azithromy- } \\
\text { cin: } 59.2\end{array}$ \\
\hline Chang D/2020 & New York/USA & Prospective (1 center) & Inpatient & 59.5 & $60.2^{\mathrm{a}}$ & 0.9 & 5.1 & $\begin{array}{l}\text { Hydroxychloroquine: } \\
\text { 56.4; Hydroxychloro- } \\
\text { quine + Azithromycin: } \\
43.6\end{array}$ \\
\hline Bhatla A/2020 & Philadelphia/USA & $\begin{array}{l}\text { Retrospective (1 } \\
\text { center) }\end{array}$ & Inpatient & 45.0 & $50.0^{a}$ & 13.0 & 11.0 & $\begin{array}{l}\text { Hydroxychloroquine: } \\
\text { 24.6; Remdesivir: } 8.1\end{array}$ \\
\hline Chorin E/2020 & New York/USA & $\begin{array}{l}\text { Retrospective ( } 2 \text { cent- } \\
\text { ers) }\end{array}$ & Inpatient & 75.0 & $64.0^{a}$ & 3.0 & 12.0 & $\begin{array}{l}\text { Hydroxychloroquine: } \\
\text { 100.0; Azithromycin: } \\
\text { 100.0 }\end{array}$ \\
\hline Sabatino J/2020 & Catanzaro/Italy & $\begin{array}{l}\text { Cross-sectional (multi- } \\
\text { center) }\end{array}$ & Inpatient & 52.6 & $34.7^{a}$ & NA & NA & NA \\
\hline Mani VR/2020 & New York/USA & $\begin{array}{l}\text { Retrospective (1 } \\
\text { center) }\end{array}$ & Inpatient & 60.3 & $64.7^{\mathrm{a}}$ & NA & 20.1 & $\begin{array}{l}\text { Hydroxychloroquine: } \\
\text { 21.7; Azithromycin: } \\
\text { 12.5; Hydroxychloro- } \\
\text { quine + Azithromycin: } \\
48.9\end{array}$ \\
\hline \multirow[t]{2}{*}{ Si D/2020 } & \multirow[t]{2}{*}{ Wuhan/China } & \multirow[t]{2}{*}{$\begin{array}{l}\text { Retrospective (1 } \\
\text { center) }\end{array}$} & Inpatient (died) & 63.6 & 64.0 & NA & 17.4 & $\begin{array}{l}\text { Azithromycin: 0.8; Anti- } \\
\text { influenza drugs: 71.9; } \\
\text { Lopinavir- Ritonavir: } \\
\text { 7.4; Remdesivir: } 0.0\end{array}$ \\
\hline & & & Inpatient (alive) & 32.7 & 61.5 & NA & 8.4 & $\begin{array}{l}\text { Azithromycin: 2.6; Anti- } \\
\text { influenza drugs: 83.7; } \\
\text { Lopinavir- Ritonavir: } \\
\text { 14.3; Remdesivir: } 2.0\end{array}$ \\
\hline Angeli F/2020 & Varese/Italy & $\begin{array}{l}\text { Retrospective (1 } \\
\text { center) }\end{array}$ & Inpatient & 72.0 & $64^{\mathrm{a}} .0$ & 6.0 & 10.0 & $\begin{array}{l}\text { Hydroxychloroquine: } \\
\text { 82.0; Macrolides: 56.0; } \\
\text { Lopinavir- Ritonavir: } \\
\text { 54.0 }\end{array}$ \\
\hline Samuel S/2020 & New York/USA & $\begin{array}{l}\text { Retrospective (1 } \\
\text { center) }\end{array}$ & Inpatient & 57.5 & $12.6^{\mathrm{a}}$ & NA & NA & $\begin{array}{l}\text { Hydroxychloroquine: } \\
\text { 44.0; Hydroxychloro- } \\
\text { quine+ Azithromycin: } \\
\text { 25.0; Remdesivir: 5.6; } \\
\text { Tocilizumab: } 5.6\end{array}$ \\
\hline Ramireddy A/2020 & Los Angeles/USA & $\begin{array}{l}\text { Retrospective (1 } \\
\text { center) }\end{array}$ & Inpatient & 61.0 & $62.3^{\mathrm{a}}$ & 20.0 & NA & $\begin{array}{l}\text { Hydroxychloroquine: } \\
\text { 10.2; Azithromycin: } \\
\text { 27.6; Hydroxychloro- } \\
\text { quine + Azithromycin: } \\
62.2\end{array}$ \\
\hline
\end{tabular}


Table 1 (continued)

\begin{tabular}{|c|c|c|c|c|c|c|c|c|}
\hline Author/Year & Country & Study design & Setting & Male & Age & $\mathrm{HF}$ & CAD & Medication \\
\hline Sala S/2020 & Milan/Italy & $\begin{array}{l}\text { Cross-sectional (multi- } \\
\text { center) }\end{array}$ & Inpatient & 66.0 & 65.0 & NA & 7.0 & $\begin{array}{l}\text { Hydroxychloroquine: } \\
\text { 100.0; Azithromycin: } \\
\text { 100.0 }\end{array}$ \\
\hline Cao B/2020 & Beijing/China & $\begin{array}{l}\text { Randomized con- } \\
\text { trolled trial (1 center) }\end{array}$ & Inpatient & 60.3 & 58.0 & NA & NA & Lopinavir-Ritonavir: 49.7 \\
\hline Goyal P/2020 & New York/USA & $\begin{array}{l}\text { Retrospective (2 cent- } \\
\text { ers) }\end{array}$ & Inpatient & 60.6 & 62.2 & NA & 13.7 & NA \\
\hline Cao J/2020 & Wuhan/China & $\begin{array}{l}\text { Retrospective (1 } \\
\text { center) }\end{array}$ & Inpatient & 52.0 & 54.0 & NA & NA & Antiviral drugs: 98.0 \\
\hline Zhang G/2020 & Wuhan/China & $\begin{array}{l}\text { Retrospective (1 } \\
\text { center) }\end{array}$ & Inpatient & 48.9 & 55.0 & NA & NA & Antiviral drugs: 88.7 \\
\hline Jun Wu/2020 & Wuhan/China & $\begin{array}{l}\text { Retrospective (1 } \\
\text { center) }\end{array}$ & Inpatient & 54.5 & 62.0 & NA & NA & Antiviral drugs: 97.0 \\
\hline $\begin{array}{l}\text { Fernández-Ruiz } \\
\text { M/2020 }\end{array}$ & Madrid/Spain & $\begin{array}{l}\text { Retrospective (1 } \\
\text { center) }\end{array}$ & Inpatient/outpatient & 77.8 & 71.0 & NA & 22.2 & $\begin{array}{l}\text { Lopinavir-Riton- } \\
\text { avir + Hydroxy- } \\
\text { chloroquine: } 44.4 ; \\
\text { Lopinavir-Ritonavir: } \\
\text { 5.6; Hydroxychloro- } \\
\text { quine: } 27.8\end{array}$ \\
\hline McCullough SA/2020 & New York/USA & $\begin{array}{l}\text { Retrospective (1 } \\
\text { center) }\end{array}$ & Inpatient & 63.2 & 64.0 & 7.3 & 14.4 & NA \\
\hline Lim JH/2020 & Daegu/Korea & $\begin{array}{l}\text { Retrospective (2 cent- } \\
\quad \text { ers) }\end{array}$ & Inpatient & 66.7 & 75.0 & 6.7 & NA & $\begin{array}{l}\text { Hydroxychloroquine: } \\
\text { 83.3; Lopinavir-Riton- } \\
\text { avir: } 96.7\end{array}$ \\
\hline Maraj I/2020 & Connecticut/USA & $\begin{array}{l}\text { Retrospective (1 } \\
\text { center) }\end{array}$ & Inpatient & 56.0 & $62.7^{a}$ & NA & 14.0 & $\begin{array}{l}\text { Hydroxychloroquine: } \\
\text { 100.0; Azithromycin: } \\
\text { 100.0 }\end{array}$ \\
\hline Shao F/2020 & Wuhan/China & $\begin{array}{l}\text { Retrospective (1 } \\
\text { center) }\end{array}$ & Inpatient & 66.2 & $69.0^{a}$ & NA & 11.0 & NA \\
\hline Lagier JC/2020 & Marseille/France & $\begin{array}{l}\text { Retrospective (multi- } \\
\text { center) }\end{array}$ & Inpatient/outpatient & 45.6 & $45.0^{a}$ & NA & NA & $\begin{array}{l}\text { Hydroxychloroquine: } \\
\text { 2.7; Azithromycin: } \\
\text { 3.7; Hydroxychloro- } \\
\text { quine + Azithromycin: } \\
89.3\end{array}$ \\
\hline Jung HY/2020 & Daegu/Korea & $\begin{array}{l}\text { Retrospective (multi- } \\
\text { center) }\end{array}$ & Inpatient & 42.9 & $63.5^{\mathrm{a}}$ & NA & NA & $\begin{array}{l}\text { Lopinavir-Ritonavir: } \\
\text { 100.0; Hydroxychloro- } \\
\text { quine: } 50.0\end{array}$ \\
\hline Dubernet A/2020 & Réunion Island/France & $\begin{array}{l}\text { Retrospective (1 } \\
\text { center) }\end{array}$ & Inpatient & 69.4 & 66.0 & NA & NA & $\begin{array}{l}\text { Hydroxychloro- } \\
\text { quine + Azithromycin: } \\
\text { 63.9; Lopinavir- Rito- } \\
\text { navir: } 5.6\end{array}$ \\
\hline Voisin O/2020 & Paris/France & $\begin{array}{l}\text { Retrospective (1 } \\
\text { center) }\end{array}$ & Inpatient & 55.2 & 68.0 & NA & NA & $\begin{array}{l}\text { Hydroxychloro- } \\
\text { quine + Azithromycin: } \\
100.0\end{array}$ \\
\hline Mazzanti A/ 2020 & Pavia/Italy & $\begin{array}{l}\text { Prospective (multi- } \\
\text { center) }\end{array}$ & Inpatient & 63.0 & 69.0 & NA & NA & $\begin{array}{l}\text { Hydroxychlo- } \\
\text { roquin:100.0; } \\
\text { Hydroxychloro- } \\
\text { quine + Azithromycin: } \\
\text { 26.0; Hydroxychlo- } \\
\text { roquin + Lopina- } \\
\text { vir- Ritonavir: } 35.0 ; \\
\text { Hydroxychloro- } \\
\text { quine + Azithro- } \\
\text { mycin + Lopinavir- } \\
\text { Ritonavir: } 6.0\end{array}$ \\
\hline Gupta MD/2020 & New Delhi/India & Case series ( 1 center) & Inpatient & 57.1 & 56.0 & 14.3 & 28.6 & NA \\
\hline Chinitz JS/2020 & New York/USA & $\begin{array}{l}\text { Retrospective (1 } \\
\text { center) }\end{array}$ & Inpatient & 42.9 & $64.0^{\mathrm{a}}$ & NA & NA & NA \\
\hline
\end{tabular}


Table 1 (continued)

\begin{tabular}{|c|c|c|c|c|c|c|c|c|}
\hline Author/Year & Country & Study design & Setting & Male & Age & $\mathrm{HF}$ & CAD & Medication \\
\hline Ferguson J/2020 & California/USA & $\begin{array}{l}\text { Retrospective (2 cent- } \\
\text { ers) }\end{array}$ & Inpatient & 52.8 & 60.4 & 6.9 & 9.7 & $\begin{array}{l}\text { Hydroxychloroquine: } \\
\text { 22.2; Azithromycin: } \\
\text { 45.8; Remdesivir: 44.4; } \\
\text { Tocilizumab: } 5.6\end{array}$ \\
\hline Argenziano MG/2020 & New York/USA & $\begin{array}{l}\text { Retrospective (1 } \\
\text { center) }\end{array}$ & Inpatient & 60.1 & 63.0 & 10.7 & 13.5 & $\begin{array}{l}\text { Hydroxychloroquine: } \\
\text { 63.9; Azithromycin: } \\
\text { 47.6; Remdesivir: 2.1; } \\
\text { Tocilizumab: } 6.0\end{array}$ \\
\hline Khamis F/2020 & Muscat/Oman & Prospective (2 centers) & Inpatient & 85.0 & $48.0^{a}$ & NA & NA & $\begin{array}{l}\text { Hydroxychloroquine/ } \\
\text { Chloroquine: 97.0; } \\
\text { Azithromycin: 71.0; } \\
\text { Lopinavir-Ritonavir: } \\
\text { 59.0; Tocilizumab: } 3.2\end{array}$ \\
\hline Russo V /2020 & Naples/ltaly & $\begin{array}{l}\text { Retrospective (multi- } \\
\text { center) }\end{array}$ & Inpatient & 61.1 & $66.9^{a}$ & 11.1 & 15.9 & NA \\
\hline Xu H/2020 & Sichuan/China & $\begin{array}{l}\text { Retrospective (1 } \\
\text { center) }\end{array}$ & Inpatient & 49.0 & NA & NA & NA & Antiviral drugs: 100.0 \\
\hline Chen L/2020 & Guangdong/China & $\begin{array}{l}\text { Retrospective (3 cent- } \\
\text { ers) }\end{array}$ & Inpatient & 67.0 & $59.5^{a}$ & NA & NA & Antiviral drugs: 96.0 \\
\hline Kelly M/2020 & Dublin/Ireland & $\begin{array}{l}\text { Retrospective (1 } \\
\text { center) }\end{array}$ & Inpatient & 61.9 & NA & NA & NA & $\begin{array}{l}\text { Hydroxychloro- } \\
\text { quine + Azithromycin: } \\
61.2\end{array}$ \\
\hline Rivinius R/2020 & Heidelberg/Germany & $\begin{array}{l}\text { Retrospective (multi- } \\
\text { center) }\end{array}$ & Inpatient & 81.0 & $58.6^{a}$ & 100.0 & NA & $\begin{array}{l}\text { Hydroxychloroquine: } \\
\text { 14.3; Azithromycin: } \\
19.0\end{array}$ \\
\hline Aversa M/2020 & New York/USA & $\begin{array}{l}\text { Retrospective (1 } \\
\text { center) }\end{array}$ & Inpatient & 50.0 & 65.0 & NA & NA & $\begin{array}{l}\text { Hydroxychloroquine: } \\
\text { 84.0; Azithromycin: } \\
\text { 75.0; Remdesivir: 9.0; } \\
\text { Tocilizumab: } 19.0\end{array}$ \\
\hline Wang ZH/2020 & Wuhan/China & $\begin{array}{l}\text { Retrospective (1 } \\
\text { center) }\end{array}$ & Inpatient & 64.4 & $67.4^{a}$ & NA & NA & $\begin{array}{l}\text { Antiviral drugs: } 88.1 ; \\
\text { Lopinavir-Ritonavir: } \\
10.2\end{array}$ \\
\hline Li J/2020 & Wuhan/China & $\begin{array}{l}\text { Retrospective (1 } \\
\text { center) }\end{array}$ & Inpatient & 47.0 & 58.0 & NA & 6.0 & Antiviral drugs: 78.4 \\
\hline Rey JR/2020 & Madrid/Spain & $\begin{array}{l}\text { Retrospective (1 } \\
\text { center) }\end{array}$ & Inpatient & 54.8 & $62.3^{a}$ & 4.9 & 6.5 & $\begin{array}{l}\text { Hydroxychloroquine: } \\
\text { 77.4; Azithromycin: } \\
\text { 45.6; Lopinavir- Riton- } \\
\text { avir: 10.4; Tocilizumab: } \\
7.4\end{array}$ \\
\hline Riker RR/2020 & Portland/USA & $\begin{array}{l}\text { Retrospective (1 } \\
\text { center) }\end{array}$ & Inpatient & 100.0 & 70.0 & 0.0 & 33.3 & $\begin{array}{l}\text { Hydroxychloroquine: } \\
\text { 66.6; Azithromycin: } \\
\text { 100.0; Remdesivir: } \\
\text { 33.3; Tocilizumab: } 33.3\end{array}$ \\
\hline Beyls C/2020 & Amiens Cedex/France & $\begin{array}{l}\text { Retrospective (1 } \\
\text { center) }\end{array}$ & Inpatient & 68.3 & NA & NA & NA & Lopinavir-Ritonavir: 100 \\
\hline Sheth V/2020 & New York/USA & $\begin{array}{l}\text { Retrospective (1 } \\
\text { center) }\end{array}$ & Inpatient & 71.0 & 69.0 & NA & NA & $\begin{array}{l}\text { Hydroxychloroquine: } \\
\text { 84.0; Azithromycin: } \\
\text { 90.0; Remdesivir: } 3.2\end{array}$ \\
\hline Ferrando C/2020 & Barcelona/Spain & $\begin{array}{l}\text { Prospective (multi- } \\
\text { center) }\end{array}$ & Inpatient & 66.8 & 64.0 & 1.4 & NA & $\begin{array}{l}\text { Hydroxychloroquine: } \\
\text { 90.1; Azithromycin: } \\
\text { 74.8; Lopinavir- Riton- } \\
\text { avir: 65.2; Remdesivir: } \\
\text { 2.9; Tocilizumab: } 42.5\end{array}$ \\
\hline Farré N/2020 & Barcelona/Spain & $\begin{array}{l}\text { Retrospective (1 } \\
\text { center) }\end{array}$ & Inpatient & 57.1 & NA & 5.3 & NA & $\begin{array}{l}\text { Hydroxychloroquine: } \\
\text { 2.6; Azithromycin: } \\
\text { 1.6; Hydroxychloro- } \\
\text { quine+ Azithromycin: } \\
\text { 93.3; Tocilizumab: } 16.9\end{array}$ \\
\hline Sridhar AR/2020 & Washington/ USA & $\begin{array}{l}\text { Retrospective (1 } \\
\text { center) }\end{array}$ & Inpatient & 60.0 & $62.0^{a}$ & 16.0 & 13.0 & $\begin{array}{l}\text { Hydroxychloroquine: } \\
100.0\end{array}$ \\
\hline
\end{tabular}


Table 1 (continued)

\begin{tabular}{|c|c|c|c|c|c|c|c|c|}
\hline Author/Year & Country & Study design & Setting & Male & Age & $\mathrm{HF}$ & CAD & Medication \\
\hline Sekhavati E/2020 & Tehran/Iran & $\begin{array}{l}\text { Randomized con- } \\
\text { trolled trial ( } 1 \text { center) }\end{array}$ & Inpatient & 50.0 & $54.3^{a}$ & NA & NA & $\begin{array}{l}\text { Azithromycin: 100.0; } \\
\text { Lopinavir-Ritonavir: } \\
100.0\end{array}$ \\
\hline Satlin MJ/2020 & New York/USA & $\begin{array}{l}\text { Retrospective ( } 2 \text { cent- } \\
\text { ers) }\end{array}$ & Inpatient & 63.0 & 62.0 & 9.0 & 18.0 & $\begin{array}{l}\text { Hydroxychloroquine: } \\
\text { 100.0; Azithromycin: } \\
\text { 18.0; Remdesivir: } 7.2\end{array}$ \\
\hline Chen L/2020 & Wuhan/China & $\begin{array}{l}\text { Retrospective (1 } \\
\text { center) }\end{array}$ & Inpatient & 76.2 & 53.0 & NA & 6.3 & Antiviral drugs: 90.5 \\
\hline Oates CP/2020 & New York/USA & $\begin{array}{l}\text { Retrospective (1 } \\
\text { center) }\end{array}$ & Inpatient & 55.0 & 69.0 & NA & 19.0 & $\begin{array}{l}\text { Hydroxychloroquine: } \\
\text { 87.0; Azithromycin: } \\
\text { 60.0; Remdesivir: 4.0; } \\
\text { Tocilizumab: } 4.0\end{array}$ \\
\hline Enzmann MO/2020 & Dakota/USA & $\begin{array}{l}\text { Retrospective (3 cent- } \\
\text { ers) }\end{array}$ & Inpatient & 56.7 & 56.0 & 10.7 & NA & $\begin{array}{l}\text { Hydroxychloroquine: } \\
\text { 6.0; Hydroxychloro- } \\
\text { quine + Azithromycin: } \\
\text { 44.0; Lopinavir-Riton- } \\
\text { avir: 2.0; Tocilizumab: } \\
\text { 8.0 }\end{array}$ \\
\hline
\end{tabular}

$C A D$ coronary artery disease, $H F$ heart failure, $N A$ not reported

a In studies not reporting the median, results are represented by the mean

myocarditis in the setting of COVID-19 [5]. Notably, higher incidence rates of conduction disorders and premature contraction were found in COVID-19 patients, compared to other types of arrhythmia, in the present study. Our findings increase clinical awareness of arrhythmia in patients hospitalized with COVID-19 for the benefit of first-line healthcare providers.

The major limitation of our study was the inclusion of studies largely from observational data with the potential risk of selection bias. For example, nearly all included studies analyzed data from inpatient settings rather than from the community, likely resulting in overestimation of the true incidence and mortality of arrhythmia among COVID-19 infections. In addition, heterogeneity within and between countries may have caused differences in the estimated incidence and clinical impacts of arrhythmia. Finally, due to the involvement of multiple factors, mortality in COVID-19 patients who developed arrhythmia cannot be entirely attributed to arrhythmia alone. However, the strength of the present study is to summarize the current evidence regarding arrhythmia and COVID-19 infection from various populations worldwide. Since COVID-19 infection probably poses increased risk of arrhythmia, significantly affecting mortality, physicians should consider arrhythmia monitoring with early management in addition to supportive care and respiratory support when treating COVID-19 patients. 


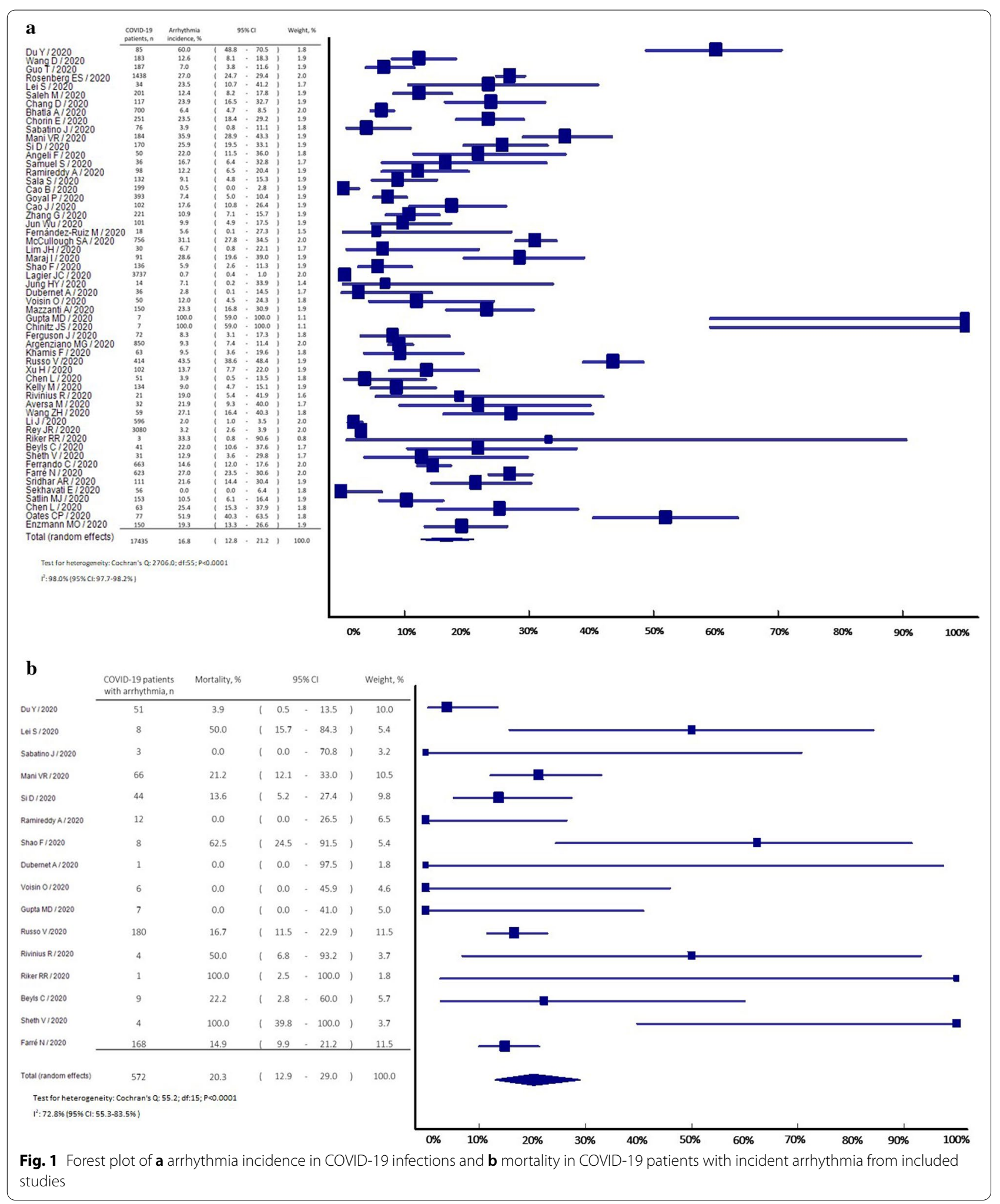




\section{Authors' contributions}

SCL and SCS contributed equally to this work. SCL and SCS performed critical analysis, interpretation of the data, and drafting of the manuscript. CWC, YCC and $\mathrm{MJH}$ contributed to study supervision and administrative, technical, or material support. All authors have read and approved the final manuscript.

\section{Funding}

This research is supported by the Chang Gung Memorial Hospital Research Project (CLRPG 2J0011).

\section{Availability of data and materials}

Not applicable.

\section{Ethics approval and consent to participate}

Not applicable.

\section{Consent for publication}

This research letter has not been published and is not under consideration by any other journal.

\section{Competing interests}

The authors declare that they have no competing interests.

\section{Author details}

${ }^{1}$ Department of Emergency Medicine, Keelung Chang Gung Memorial Hospital, Keelung, Taiwan. ${ }^{2}$ College of Medicine, Chang Gung University, Taoyuan, Taiwan. ${ }^{3}$ School of Pharmacy, Institute of Clinical Pharmacy and Pharmaceutical Sciences, College of Medicine, National Cheng Kung University, Tainan, Taiwan. ${ }^{4}$ Department of Pharmacy, Keelung Chang Gung Memorial Hospital, Keelung, Taiwan. ${ }^{5}$ Section of Cardiology, Department of Internal Medicine, Keelung Chang Gung Memorial Hospital, No. 222, Maijin Rd., Anle Dist.
Keelung, Taiwan. ${ }^{6}$ Section of Nephrology, Department of Internal Medicine, Linkou Chang Gung Memorial Hospital, Taoyuan, Taiwan. ${ }^{7}$ Community Medicine Research Center, Keelung Chang Gung Memorial Hospital, Keelung, Taiwan.

Received: 30 September 2020 Accepted: 3 November 2020

Published online: 10 December 2020

\section{References}

1. Kang Y, Chen T, Mui D, et al. Cardiovascular manifestations and treatment considerations in covid-19. Heart. 2020;106(15):1132-41.

2. Wang D, Hu B, Hu C, et al. Clinical characteristics of 138 hospitalized patients with 2019 novel coronavirus-infected pneumonia in Wuhan China. JAMA. 2020;323(11):1061-9.

3. Guo T, Fan Y, Chen M, et al. Cardiovascular implications of fatal outcomes of patients with coronavirus disease 2019 (COVID-19). JAMA Cardiol. 2020;5(7):1-8.

4. Corrales-Medina VF, Suh KN, Rose G, et al. Cardiac complications in patients with community-acquired pneumonia: a systematic review and meta-analysis of observational studies. PLoS Med. 2011:8(6):e1001048.

5. Driggin E, Madhavan MV, Bikdeli B, et al. Cardiovascular considerations for patients, health care workers, and health systems during the COVID-19 pandemic. J Am Coll Cardiol. 2020;75(18):2352-71.

\section{Publisher's Note}

Springer Nature remains neutral with regard to jurisdictional claims in published maps and institutional affiliations.
Ready to submit your research? Choose BMC and benefit from:

- fast, convenient online submission

- thorough peer review by experienced researchers in your field

- rapid publication on acceptance

- support for research data, including large and complex data types

- gold Open Access which fosters wider collaboration and increased citations

- maximum visibility for your research: over $100 \mathrm{M}$ website views per year

At BMC, research is always in progress.

Learn more biomedcentral.com/submissions 